\title{
Odontostomatological manifestations in sickle cell disease: A systematic review
}

\section{Objawy stomatologiczne w niedokrwistości sierpowatej - przegląd systematyczny}

\author{
Gianfilippo Nifosi ${ }^{1, A-D, F}$, Antonio Fabrizio Nifosi ${ }^{2, B, D-F}$, Lorenzo Nifosi ${ }^{3, B, D-F}$ \\ 1 CHU Brugmann, Bruxelles, Belgium \\ 2 Dental Clinic iDental, Valencia, Spain \\ ${ }^{3}$ European University of Valencia, Valencia, Spain \\ A - research concept and design; $\mathrm{B}$ - collection and/or assembly of data; $\mathrm{C}$ - data analysis and interpretation; \\ $D$ - writing the article; $E$ - critical revision of the article; $F$ - final approval of article
}

Address for correspondence

Gianfilippo Nifosi

E-mail: g.nifosi@tin.it

Funding sources

none declared

Conflict of interest

none declared

Received on March 10, 2017

Revised on June 24, 2017

Accepted on July 10, 2017

DOI

$10.17219 / \mathrm{dmp} / 75796$

Copyright

() 2017 by Wroclaw Medical University

and Polish Dental Society

This is an article distributed under the terms of the

Creative Commons Attribution Non-Commercial License

(http://creativecommons.org/licenses/by-nc-nd/4.0/)

\begin{abstract}
Sickle-cell disease is a frequent hemoglobinopathy which represents a major public health problem as a result of the continuous migration of populations. The clinical manifestations are chronic hemolytic anemia, vaso-occlusive crisis and infections; however, the involvement of the head and neck region remains poorly reported. Particularly, we do not know the prevalence of the odontostomatological manifestations.

In this study, we reviewed the most recent literature on the topic. We have classified the alterations of the orofacial sphere into four categories: oral manifestations, bone changes, infections and various. We have described the main clinical manifestations of each one, along with their incidence and prevalence, trying to identify the pathophysiological mechanisms, correlations with vaso-occlusive crisis and possible therapeutic strategies. Several odontostomatological manifestations are described in patients with sickle cell disease. Some are typical, while others still need to be investigated, as for example the relationship between oral triggers and vaso-occlusive crisis.
\end{abstract}

Key words: sickle cell anemia, bone marrow hyperplasia, orofacial alterations

Słowa kluczowe: niedokrwistość sierpowata, rozrost szpiku kostnego, zmiany w jamie ustnej 
Sickle cell disease (SCD) is an hereditary blood disease characterized by the production of abnormal hemoglobin, known as $\mathrm{Hb} S$, characterized by a point mutation in the $6^{\text {th }}$ codon of exon 1 of the $\beta$-globin gene resulting in the replacement of the regular glutamic acid with valine at position 6 in the $\mathrm{NH}_{2}$ terminal end of the $\beta$-globin chain. Other types of SCD syndromes are characterized by double heterozygous $\beta S / \beta C, \beta S / \beta+$ thal, $\beta S / \beta 0$ thal and $\beta S / \beta X$ $(\mathrm{D}, \mathrm{E}$, etc.). The abnormality characterizing $\mathrm{Hb} \mathrm{C}$ has to do with the replacement of the normal glutamic acid with lysine at position 6 of the $\beta$-globin chain. In conditions of reduced partial oxygen tension, the unstable $\mathrm{Hb} \mathrm{S}$ polymerizes, forming fluid polymers (tactoids) that deform blood red cells in a shape characteristic of reversible sickle that may plug different areas of the microcirculation or a large vessel, potentially causing vascular ischemic damage in many organs. SCD is most common in sub-Saharan African people, Asian, Arabia, and Mediterranean countries. Across equatorial Africa, the prevalence of SCD trait carriers ranges between 10 and 40\%. Approximately 2\% of births in Ghana and Nigeria have this type of disease. As a result of migration, this has become a public health problem, both in the Western and developing countries. Epidemiological studies have shown that $7 \%$ of the world population is a carrier of a hemoglobin variant, while about 300,000-400,000 newborns each year are affected by serious hemoglobinopathies: among these, sickle cell anemia is the most common.

Clinically, 2 phenotypes can be distinguished: 1 . hemolysis-associated, characterized by anemia, leg ulcers and pulmonary hypertension; 2. vaso-occlusal, characterized by acute chest pain, stroke and avascular necrosis of joints. SCD crises are still responsible for high morbidity and early mortality. The hospitalization is very frequent in pain vaso-occlusive crises. Early analgesic treatment and overhydration are necessary. Severe acute crises are characterized by end-organ ischemia and infarction combined with the downstream effects of hemolysis that result from red blood cell sickling. Acute chest syndrome is a vaso-occlusive crisis of the pulmonary circulation that can be associated with one or more symptoms, including fever, cough, excruciating pain, sputum production, shortness of breath, or low oxygen levels and eventually associated also to pulmonary infiltrate on a chest $\mathrm{X}$-ray. It represents the second cause of hospitalization for SCD patients and one of the major causes of mortality. More severe cases may need treatment in an intensive care unit. An SCD patient has an increased risk of approx. 300 times of symptomatic stroke compared to a healthy patient. It is ischemic in $85 \%$ of the cases. The cumulative risk is $11 \%$ within 18 years old and $24 \%$ within 45 years old. We recommend an automatic or a manual erythroaferesis with a target of $\mathrm{Hb} 10 \mathrm{~g} / \mathrm{dL}$ and $\mathrm{Hb} \mathrm{S}<30 \%$, if $\mathrm{Hb}<9 \mathrm{~g} / \mathrm{dL}$ urgently performs a simple transfusion with a target of $\mathrm{Hb} 10 \mathrm{~g} / \mathrm{dL}$. Doppler transcranial identification of high-risk patients provided primary stroke pre- vention in SCD children. Aplastic crises may be caused by several types of infections, some viral, and especially ones including the most frequent Parvovirus B19. The diagnosis is serological and the treatment is transfusional. Sequestration syndromes are caused by the trapping and destruction of red blood cells species within the splenic and liver sinuses. Sequestration in a baby is usually splenic, wheras in an adult it is more frequently hepatic. Mortality is high and recurrence affects more than half of the patients. Infections are the leading cause of death, especially in children. They can be the trigger cause but also the evolution of the typical complications of the disease. The most common infections are: bacteremia, sepsis and meningitis caused by Streptococcus pneumoniae, Hemophilus influenzae and Neisseria meningitides; ACS caused by Streptococcus pneumoniae, Mycoplasma pneumoniae and Chlamydia pneumoniae; osteomyelitis and/ or septic arthritis caused by Salmonella spp, Staphylococcus aureus and Streptococcus pneumoniae. Penicillin oral prophylaxis and vaccinations need to be provided to children within their first 6 years of life. Several chronic complications are described: ischemic complications, eye disorders, avascular necrosis, priapism, anemic complications, heart disease, pulmonary disease, chronic kidney disease, endocrine/nutritional/metabolic disorders, chronic legs ulcers and infections. Some organs are targets of acute or chronic manifestations due to their peculiar anatomical and functional organization. Given the recurrent nature of these events, over time chronic alterations of many organs develop, that are very disabling and worsening the quality of life of these young patients. Involvement of the head and neck region remains poorly reported. SCD can involve the oral cavity, inner ears, orbits, paranasal sinuses, bones, lymph nodes and vessels. When it occurs, the basic pathogenicity is similar to that of other organs.

Life expectancy varies according to the different genotypes and geographic area. In the 1990s the average age of death was 42 years for males and 48 years for females in the SS genotype and 60 years for males and 68 years for females in the SC genotype. ${ }^{1}$ Recent literature on the subject is lacking, but life expectancy has certainly improved in countries with more developed health systems that can ensure better care for these patients.

Hydroxyurea, transfusions, iron chelation therapy and, when indicated, bone marrow transplantation are fundamental pillars of the treatment.

\section{Objectives}

The aim of this study was to review the literature on pathological manifestations of odontostomatological interest in SCD, describe their pathogenetic mechanism and correlation with the occurrence of the sickle cell crisis and to identify appropriate early intervention strategies. 
Systematic critical review of the clinical studies and case reports published on the topic in Pubmed, Google Scholar and Lilacs electronic databases, using the following keywords: "orofacial alterations in sickle cell disease" and "odontostomatological manifestations in sickle cell anemia".

Table 1 presents the classification of odontostomatological pathological manifestations in SCD most frequently encountered in the literature.

\section{Oral manifestations}

There are various oral complications that have been studied for some time.

\section{Soft tissue signs}

Mucosal pallor, yellow tissue coloration and smooth tongue for atrophy of lingual papillae are suggestive of disease. Gingival enlargement and bleeding, when present in this type of patients, especially in adolescents, can lead to the inflammation of the gums, caused by malocclusion or bad oral hygiene. Scrapings obtained from the oral tissues showed the presence of iron deposition in $35 \%$ of homozygous patients (SS), and less than $35 \%$ in patients with $B$-thalassemia, who usually undergo more blood transfusions, suggesting that iron overload may be responsible for dental complications. ${ }^{2}$

\section{Dental alterations}

More than $60 \%$ of SCD patients present intrinsic opacity of the teeth, linked to hypomaturation and hypomineralization of enamel and dentin. Erosion of enamel, more rarely of dentin, results from the acidic activity on

Table 1. Odontostomatological manifestations in SCD

\begin{tabular}{l} 
A) Oral manifestations \\
- soft tissue signs \\
- dental alterations \\
- pulp necrosis \\
\hline B) Bone changes \\
- changes in the craniofacial bones \\
- malocclusion \\
- temporomandibular joint disorders \\
- osteoporosis and osteosclerosis \\
C) Infections \\
- osteomyelitis of the jaw \\
- dental infections and sickle cell crisis \\
\hline D) Various \\
\hline - mental nerve neuropathy \\
- orofacial pain \\
- idhiopatic facial swelling
\end{tabular}

dental tissue. This occurs most often in children drinking a lot of fruit juices or in adults with certain disorders such as gastroesophageal reflux. Without correct oral hygiene, mainly based on fluorine, the hypomineralisation of the enamel can lead to serious alterations of the dental tissues. Infractions are micro-cracks within the enamel, reported anecdotally. Hypodontia has been described in one patient with $\mathrm{Hb} \mathrm{SC}$ disease. ${ }^{3}$ Delays in dental eruption are commonly reported in SCD patients.

Although there is no direct evidence that SCD causes the appearance of caries, there are numerous papers which indicate that the state of oral health in these patients worsens due to the socioeconomic status, the family income or the deficiency in oral hygiene, being a negative factor the life in houses that offer little space to children and adolescents. ${ }^{4}$ The relationship between father's education with regard to oral hygiene and the incidence of decay of teeth in children was not reported as significant. ${ }^{5}$ Conversely, it has been observed on a large number of family members, race and religion, that there is a higher incidence of tooth decay in the black population and among unbelievers. Frequent consumption of soft drinks containing sugar for hydration and inadequate oral hygiene are predisposing factors. ${ }^{6}$ It is possible that the biofilm matrix of C. albicans isolated from the saliva of SS children presented a particular caries-associated virulence for the great quantity of acids and extracellular polysaccharides and proteins. ${ }^{7}$ Moreover, penicillin prophylaxis prevents the acquisition of Streptococcus mutans, both of which may promote the growth of Candida spp. actively involved in the demineralization of dental tissues. Also, reference should be made to the state of oral health worsening, due to the various VOCs presented by these patients, since it weakens them and at the same time limits their ability to cope. SCD patients have increased susceptibility to caries, with a higher prevalence of tooth decay and a lower prevalence of filled teeth. Finally, the severity of the caries is related to age, the number of hospitalizations and the need for transfusions or drugs.

The literature agrees that SCD does not appear to predispose to periodontal disease. ${ }^{8}$ The main predisposing factors for periodontal pockets are older age and not using dental floss on a daily basis. Few reports report an association between SCD and periodontal inflammation, hypothesizing a causal link between repeated episodes of hemorrhage with secondary fibrosis and gingival enlargement. ${ }^{9}$ Nevertheless, the role of periodontal infections, which trigger of VOCs, is unknown.

\section{Pulp necrosis}

Pulp necrosis (PN) is an acute or chronic inflammation of the dental pulp as a result of deep caries, including the destruction of the microvascular and lymphatic system 
and, ultimately, the nerve fibers. It is caused by caries, trauma, bruxism and the development defect of dentine. It has been demonstrated that the frequency of asymptomatic pulp necrosis in patients with SCD is considerable. Costa et al. observed that PN was present in 50 patients (80.64\%) with SCD. ${ }^{10}$ The dental groups with the highest means of teeth with $\mathrm{PN}$ were the premolars and incisors in both the SCD group and the control group. The number of teeth with PN was higher in the exposed group for canines, premolars, and molars. Pathogenetically, PN may be due to the sikling that causes vasocclusion within the microcirculation of the dental pulp. Infarction/thrombosis of pulp vessels may expose the vital pulp to hypoxia and persistent hypoxia may cause necrosis that radiologically appears as a periapical lesion. ${ }^{11}$ Sickle-shaped cells in the dental pulp vessels can be found 2 to 3 days after an acute crisis. ${ }^{12}$ Some SCD patients may remain asymptomatic to pulp changes. Correct oral hygiene prevents caries and, consequently, the necrosis of dental pulp.

\section{Bone changes}

\section{Changes in the craniofacial bones}

Systemic manifestations include oral and craniofacial disorders, such as frontal bossing, maxillary protrusion, with retrusion of the upper and lower incisors, typical of this disease. The pathogenic explanation for this disease is found in red blood cells. The lifespan of these cells is reduced to 10-20 days and needs constant replacement and production. For this reason, many patients present compensatory bone marrow hyperplasia, which results in craniofacial bone changes, ${ }^{13}$ such as nasal septum depression, maxillary protrusion and formation of osseous trabeculae. Other oral manifestations include bone alterations such as osteoporosis of the alveolar ridge and mandibular overgrowth.

\section{Malocclusion}

The structural changes at the bone level, discussed above, promotes the development of dental malocclu$\operatorname{sion}^{14}(\mathrm{MO})$, abnormalities in the teeth, which causes a certain degree of aesthetic discomfort and functional disorders or disabilities. Craniofacial abnormalities are considered factors that contribute to the development of MO. The most common MO is Angle's Class II. Dental and functional deviations produce changes in swallowing, chewing, salivation and phonation. Additionally, a psychosocial disorder may diminish interpersonal relationships. Luna et al. reports a prevalence of MO in preschool children of $62.9 \%$, with $37.1 \%$ class II, $28.6 \%$ increased overjet, $28.6 \%$ reduced overbite and $17.1 \%$ open bite. ${ }^{15}$ The same study explains that adulthood maxillary protrusion varies between 21 and 88\%, and deep overbite between 30 and $80 \%$. SCD and MO are considered public health problems.
This suggests the need to establish a public health policy to develop community programs, rapid diagnosis, proper treatment of malocclusion and the delay of dental eruption in this population, to improve quality of life of these people. The dental MO is not exclusive of SCD, but is frequent in many systemic diseases involving skeletal alterations. In a study by Licciardello et al., it was observed that cephalometric values indicated a posterior rotation of the mandible and an increased total facial height. ${ }^{16}$ This deviation was found in all genotypes of the disease, directing the patients towards a dolichofacial pattern. In the same study, the maxillary incisors were significantly inclined towards the buccal, whereas the position of the maxillary first molar to PTV line was significantly increased only in $ß S$, ßTh patterns. Cephalometric results indicate a trend toward a vertical or dolichofacial pattern.

\section{Orthodontic treatment}

The treatment in these cases is based on using orthodontics to change the class II and regulate the levels of protrusion/retrusion. In most cases, the first upper premolars are removed to create space and thus reduce the protrusion. Both with perfect hygiene, and a year of orthodontics, with retainer at the end of treatment, a significant change is achieved at a functional, esthetic and psychological level in these patients. Of course, oral hygiene plays a fundamental role in orthodontic treatment, since oral infections are considered an added risk factor in this disease. ${ }^{1}$

\section{Temporomandibular joint disorders}

SCD patients are particularly susceptible to joint complications during the VOCs. The temporomandibular joint (TMJ) is not immune from this involvement, although there are few papers in the literature about TMJ disease, mainly case reports. Adolescents are especially susceptible to mandibular condylar osteonecrosis, preceded by an extensive and long-lasting edema in the same area, due to the bone overgrowth. This alteration is not exclusive of this disease, but common in many other systemic disorders or it may appear as a single alteration of a systemic disease. A conservative approach is essential to prevent the development of irreversible TMJ deformities. ${ }^{18}$

\section{Osteoporosis and osteosclerosis}

Abnormal radiological patterns have been found in patients with SCD. Abnormal projections of the skull, vertical trabeculations, diplopic space, and a granular appearance of the skull were found. Conditions of mandibular expansion, hyperplasia, widening of the bone marrow and thickening of the bone trabecoles were also found.

In general, the main radiographic changes found in these patients can be classified into: 1 . Radiolucent lesions 
between the root apex of posterior teeth and the mandibular inferior border, associated with osteoporotic lesions, derived from compensatory bone marrow hyperplasia. ${ }^{19}$ These lesions create in some cases a horizontal trabecular pattern described as «step-ladder», related to the presence of hemolytic anemia. 2. Radiopaque lesions associated to the bone infarction and osteonecrosis and mainly in the mandibular posterior region, coinciding with severe facial pain during VOCs. 3. Osteomyelitis lesions associated to the periapical abscess or pericoronitis.

\section{Infections}

\section{Osteomyelitis of the jaw}

Osteomyelitis is an inflammatory condition of the bone that begins in the medullary cavity and extends to the overlying periosteum. It is more common in long bones. In jaw bones, it is very rare, but when present, it resides more commonly in the posterior area of the mandible. This is explained by the lower blood flow in the jaw. Incidence of this amounts to $5 \%$ of all the osteomyelitis of the jaw: there is a predominance of this disease among men (81\%) and young people. Streptococcus and Salmonella are more frequently responsible for it. Microorganisms can infect the bone through in 3 ways: bloodstream, contiguously from local area of infection and trauma or iatrogenic causes such as periapical periodontitis in the teeth. ${ }^{20}$ It is interesting to note that SCD patients can have ostemyelitis more frequently than other patients without SCD, most likely due to the sickling and proinflammatory state reducing interleukin-10 secretion. ${ }^{21}$ Radiological aspects of indistinct bone radiolucency can pose problems of differential diagnosis with periapical cystis and keratocystic odontogenic tumors. ${ }^{22}$ Good oral hygiene could prevent oral infections and could have a preventive role in the development of bone lesions, but it is unknown whether these lesions may also occur alongside perfect oral hygiene, due to its possible genetic etiology.

\section{Dental infections and sickle cell crisis}

The chronic nature of SCD, concurrently with frequent hospital admissions for pain crisis, can affect the psychosocial sphere and reduce quality of life. Generally, in many parts of the United States, not enough attention is paid to SCD and oral health in public health systems, despite SCD being related with low oral health like increased risk of dental caries and pulp necrosis. Rada et al. suggest that dental infections in patients with SCD can produce a sickle cell crisis event and, consequently, an increased likelihood of hospital admission. ${ }^{23}$ Specifically, an interesting North American study from the Nationwide Emergency Department Sample (NEDS), between 2006 and 2008, which includes 28 million ED visits at 980 hospitals every year reported that the dental infection was related with a higher probability for SCD patients being admitted following a visit (72\% more likely than without SCD), especially in females. ${ }^{24}$ In regards to this, it is also important to keep in mind that the probability is as important as the established clinical variables, such as the avascular necrosis $(79 \%)$ and pneumonia (72\%). An inflammatory state caused by a dental infection can trigger or precipitate a sickle crisis. This higher relationship should lead to a reflection about the public health system and the integration of better prevention and dental care in primary care.

\section{Various}

\section{Mental nerve neuropathy}

The SSD is an important systemic cause of mental nerve neuropathy or Numb Chin Syndrome (NCS). According to Mendes et al., its prevalence is 2.2 times higher than observed in normal individuals. ${ }^{25}$ More frequent in females and almost always unilateral, it appears along with anesthesia or ipoesthesia of the chin and lower lip. The inferior alveolar nerve can be compromised. The particular anatomical arrangement of the nerve, confined within the relatively long bone mandibular canal, explains the higher risk for ischemic damage during a sickle cell crisis. ${ }^{26}$ In a different diagnosis, all the causes have been indicated: infections, neoplasms, trauma, dental anesthesia and other systemic diseases.

\section{Orofacial pain}

SCD patients may present symptomatic orofacial pain due to a specific oral and dental pathology including dental caries, enamel hypomineralization, pulp necrosis, and infections. Idiopathic mandibular or maxillary pain has a high prevalence in SCD that affects $49 \%$ of Jamaican patients. ${ }^{27}$ A detailed study in a large cohort of patients is lacking in the literature. A possible pathological mechanism is the sickling of the red cells within the microcirculation of the dental pulp and facial bones, ${ }^{27}$ causing small microinfarcts, favored by local or systemic conditions of increased viscosity.

The prevalence of recurrent headaches among SCD patients is unrecognized. Recently, a high prevalence has been reported, especially in children and adolescents (24.0$43.9 \%) .{ }^{28}$ An acute attack requires careful assessment to exclude several potentially devastating conditions including vascular disease, bone infarcts, tooth infections, and osteomyelitis. There is no data of headache prevalence among adults, who are at a greatest risk of medication overuse for headaches, secondary to frequent opioid use.

\section{Idhiopatic facial swelling}

Often in young people, the bone marrow hyperplasia following extramedullary hematopoiesis may lead to the 
occurrence of tender swelling, without evident causes. Several sites have been described: orbit, paranasal sinus, ${ }^{29}$ middle ear, ${ }^{30}$ thyroid, and cervical lymph nodes. Lastly, subperiosteal orbital hematoma may be secondary to the extravasation of blood from necrotic vessel walls during bright VOCs, underlying bleeding diathesis and minor trauma. ${ }^{31,32}$ The differential diagnosis must include all infections and cancer. ${ }^{33}$ These last alterations are not exclusive of SCD, but are frequent in many other systemic diseases, or they may appear as a single alteration of a systemic disease.

\section{Conclusions}

SCD is characterized by special oral health findings, whose prevalence and clinical impact are not well know. They depend not only on the presence of sickle disease, but also on subject-related factors such as oral hygiene, diet habits and social conditions. SCD does not seem to be a risk factor for conditions such as caries and periodontal diseases. Conversely, there are other typical manifestations, such as soft tissue signs and bone changes. SCD appears to be a risk factor for moderate and very severe malocclusion and orofacial pain. The relationship between the oral infectious focus and the vaso-occlusive crisis is further explained by means of studies of large samples of population. It is recommended that periodic dental screening should be part of the general physical examination. Regular oral hygiene maintenance could help prevent many potentially serious complications, contributing to the maintenance of the physical well-being of these patients.

\section{References}

1. Platt OS, Brambilla DJ, Rosse WF, et al. Mortality in sickle cell disease - life expectancy and risk factors for early death. N Engl J Med. 1994;330:1639-1644.

2. Singh J, Singh N, Kumar A, et al. Dental and periodontal health status of Beta talassemia maior and sickle cell anemia patients: A comparative study. J Int Oral Health, 2013;5:53-58.

3. Oredugba FA. Hypodontia in an adolescent with the HbSC genotype: A case report. Int J Paedriat Dent. 2005;15:455-458.

4. Luna ACA, Rodrigues MJ, Menezes VA, et al. Caries prevalence and socioeconomic factors in children with sickle cell anemia. Braz Oral Res. 2012;26:43-49.

5. Matta Felisberto Fernandes ML, et al. Caries prevalence and impact on oral health-related quality of life in children with sickle cell disease: Cross-sectional study. BMC Oral Health, 2015;15:68.

6. Ballas SK, de Castro Lobo CL, Cavalcanti WE. Dental complications of sickle cell disease. J Interdiscipl Med Dent Sci. 2014;26:1000152.

7. Brighenti FL, Medeiros AC, Matos BM, et al. Evaluation of cariesassociated virulence of biofilms from Candida albicans isolated from saliva of paediatric patients with sickle-cell anemia. J Appl Oral Sci. 2014;22:484-489.

8. Al-Alawi H, Al-Jawad A, Al-Shayeb M, et al. The association between dental and periodontal diseases and sickle cell disease. A pilote case-control study. Saudi Dent J. 2015;27:40-43.

9. Scipio JE, Al-Bayaty HF, Murti PR, et al. Facial swelling and gingival enlargement in a patient with sickle cell disease. Oral Dis. 2001;7:306-309.
10. Costa CP, Thomaz EB, Souza Sde F. Association between sickle cell anemia and pulp necrosis. J Endod. 2013;39:177-181.

11. Andrews $\mathrm{CH}$, England MC Jr, Kemp WB. Sickle cell anemia: An etiological factor in pulpal necrosis. J Endod. 1983;9:249-252.

12. Cox GM, Soni NN. Pathological effects of sickle cell anemia on the pulp. ASDC J Dent Child. 1984;51:128-132.

13. Fernandez M, Slovis TL, Whitten-Shurney W. Maxillary sinus marrow hyperplasia in sickle cell anemia. Pediatr Radiol. 1995;25(suppl 1): S209-211.

14. Costa CPS, Correia de Carvalho HLC, Fonseca Thomaz EBA. Is sickle cell anemia a risk factor for severe dental malocclusion? Braz Oral Res. 2015;29:1-7.

15. Luna AC, Godoy F, de Menezes VA. Malocclusion and treatment need in children and adolescent with sicle cell disease. Angle Orthod. 2014;84:467-472.

16. Licciardello V, Bertuna G, Samperi P. Craniofacial morphology in patients with sicle cell disease: A cefalometric analysis. Eur J Orthod. 2009;29:238-242.

17. Amoah KG, Newman-Nartey M, Ekem I. The orthodontic management of an adult with sicle cell disease. Ghana Med J. 2015;49:214-218.

18. Baykul T, Aydin MA, Nasir S. Avascular necrosis of the mandibular condyle causing fibrous ankylosis of the temporomandibular joint in sickle cell anemia. J Craniofac Surg. 2004;15:1052-1056.

19. Avsever IH, Orhan $\mathrm{K}$, Özen $\mathrm{T}$, et al. Evaluation of mandibular bone structure in sickle cell anemia patients. Güihane Tip Derg. 2015:57:11-15.

20. Olaitan AA, Amuda JT, Adekeye EO. Osteomyelitis of the mandible in sickle cell disease. Br J Oral Maxillofac Surg. 1997;35:190-192.

21. Sarray S, Almawi WY. Contribution of reduced interleukin-10 levels to the pathogenesis of osteomyelitis in children with sickle cell disease. Clin Vaccine Immunol. 2015;22:1020-1024.

22. Araújo JP, Cadavid $A M H$, Lemos $C A$, et al. Bilateral mandibular osteomyelitis mimicking periapical cysts in a patient with sickle cell anemia. Autopsy Case Rep. 2015;5:55-60.

23. Rada RE, Bronny AT, Hasiakos PS. Sickle cell crisis precipitated by periodontal infection: Report of two cases. J Am Dent Assoc. 1987;114:799-801.

24. Laurence B, Haywood C Jr, Lanzkron S. Dental infections increase the likelihood of hospital admissions among adult patients with sickle cell disease. Community Dent Health. 2013;30:168-172.

25. Bar-Ziv J, Slasky BS. CT imaging of mental nerve neuropathy: The numb chin syndrome. Am J Roentgenol. 1997;168:371-376.

26. O'Rourke CA, Hawley GM. Sickle cell disorder and orofacial pain in Jamaican patients. Br Dent J. 1998;185:90-92.

27. O'Rourke C, Mitropoulos C. Orofacial pain in patients with sickle cell disease. Br Dent J. 1990;169:130-132.

28. Vgontzas A, Charleston L, Robbins M. Headache and facial pain in sickle cell disease. Curr Pain Headache Rep. 2016;20:20.

29. Collins WO, Younis RT, Garcia MT. Extramedullary hematopoiesis of the paranasal sinuses in sickle cell disease. Otolaryngol Head Neck Surg. 2005;132:954-956.

30. Applebaum EL, Frankel A. Extramedullary hematopoiesis of the middle ear. Am J Otolaryngol. 1989;10:287-290.

31. Ganesh A, Al-Zuhaibi S, Pathare A, et al. Orbital infarction in sickle cell disease. Am J Ophthalmol. 2008;146:595-601.

32. Procianov F, Brandão FM, Cruz AA, et al. Subperiosteal hematoma and orbital compression syndrome following minor trauma in sickle cell anemia: Case report. Arq Bras Oftalmol. 2008;71:262-264.

33. Swapnil M, Ajay P, Kanishka NG, et al. Idhiopatic facial swelling secondary to sickle cell anemia. BMJ Case Rep. 2012;2012:201200713. 